\title{
Unexpected tachycardia and hypertension during anesthetic induction with remimazolam in cardiac surgery: a case report
}

Tomoe Sato, Sho Ohno, Makishi Maeda, Yasuaki Sawashita, Naoyuki Hirata* ${ }^{*}$ and Michiaki Yamakage

Keywords: Remimazolam, Cardiac surgery, Adrenergic response

To the Editor

Remimazolam, which is a short-acting benzodiazepine, has been available for general anesthesia in Japan $[1,2]$. Previous clinical trials showed that the incident rate of intraoperative hypotension during general anesthesia with remimazolam was lower than that with propofol, while the efficacy of general anesthesia with remimazolam and that with propofol in non-cardiac surgery were comparable [1]. Thus, the effects of remimazolam on hemodynamics may be different from those of existing anesthetics. In this case report, we describe a case in which unexpected tachycardia and hypertension occurred during anesthetic induction using remimazolam in cardiac anesthesia.

\section{Case presentation}

A 53-year-old man $(161 \mathrm{~cm}, 83.6 \mathrm{~kg})$ who had severe aortic valve stenosis and regurgitation due to a bicuspid aortic valve was scheduled for aortic valve replacement and ascending aortic replacement. He had no history and was not taking any medication.

Baseline blood pressure (BP) and heart rate (HR) were $143 / 90 \mathrm{mmHg}$ and $74 \mathrm{bpm}$ with sinus rhythm, respectively. General anesthesia was induced by $6 \mathrm{mg} /$ $\mathrm{kg} / \mathrm{h}$ remimazolam, $100 \mu \mathrm{g}$ fentanyl, and $0.15 \mu \mathrm{g} / \mathrm{kg} /$ min remifentanil. After loss of consciousness, the dose of remimazolam was decreased to $1 \mathrm{mg} / \mathrm{kg} / \mathrm{h}$ and

*Correspondence: naohirata@mac.com

Department of Anesthesiology, Sapporo Medical University School

of Medicine, South 1, West 16, Chuo-ku, Sapporo, Hokkaido 060-8543, Japan rocuronium $(0.8 \mathrm{mg} / \mathrm{kg})$ was administered. Although manual mask ventilation was performed without difficulty and BIS value was 46 to 62 during ventilation, HR gradually increased from 72 to $103 \mathrm{bpm}$, and BP also increased from $115 / 59 \mathrm{mmHg}$ to $157 / 86 \mathrm{mmHg}$ (Fig. 1). At that time, end-tidal carbon dioxide concentration was $35-40 \mathrm{mmHg}$, and there was no problem with the intravenous line for administration of remimazolam (e.g., occlusion, formation of a precipitation, extravasation). We discontinued remimazolam and started administration of sevoflurane, suspecting that remimazolam was responsible for adrenergic responses. After the end-tidal sevoflurane concentration reached $1.5 \%, \mathrm{HR}$ and $\mathrm{BP}$ decreased to $84 \mathrm{bpm}$ and $115 / 61 \mathrm{mmHg}$, respectively, and the BIS value was 46 . The patient was intubated about 7 min after administration of rocuronium. After the anesthetic agent had been changed from remimazolam to sevoflurane during anesthetic induction and propofol was administered during cardiopulmonary bypass, there were no unexpected hemodynamic changes throughout the anesthetic period. Surgical procedures were completed successfully. There was no peri-anesthetic awareness and/or recall that could have induced adrenergic responses.

\section{Discussion and conclusions}

We experienced unexpected tachycardia and hypertension during anesthetic induction using remimazolam in a cardiac surgical patient, although remimazolam was used according to the instructions in the package insert.

We believe that intraoperative awareness did not occur in this case because BIS values were appropriate during 


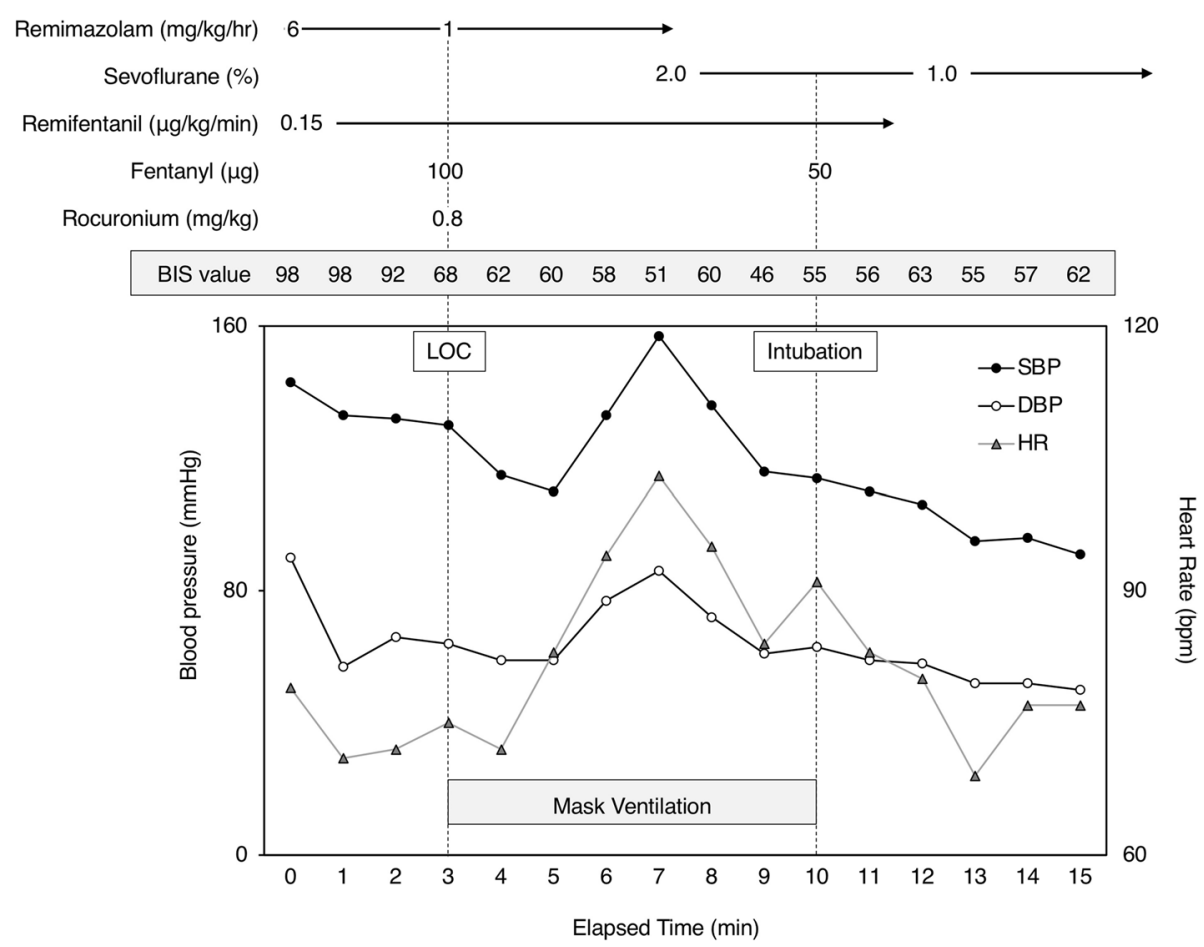

Fig. 1 Anesthetic chart during anesthetic induction. LOC, loss of consciousness; BIS, bispectral index; SBP, systolic blood pressure; DBP, diastolic blood pressure; $H R$, heart rate

the occurrence of adrenergic responses. However, the validity of BIS in anesthesia with benzodiazepines has been controversial [3, 4]. Therefore, intraoperative awareness without recall or agitation via inadequate anesthetic depth might have induced the adrenergic responses. Other factors including hypercapnia, stress of manual mask ventilation, and/or the effects of remimazolam on the autonomic nervous system might also have contributed to the adrenergic responses in this case.

Further accumulation of clinical studies and reports on remimazolam would be desirable to increase the clinical reliability of remimazolam for general anesthesia.

\section{Abbreviations}

BP: Blood pressure; HR: Heart rate; BIS: Bispectral index.

\section{Acknowledgements}

Not applicable.

\section{Authors' contributions}

ST, OS, MM, SY, and NH performed anesthetic management for the patient. ST, $\mathrm{NH}$, and YM drafted the manuscript. All authors have read and approved the final manuscript.

\section{Funding}

The authors have not received money, gifts, or other compensation from any organization, institution, or business that may be affected financially by our publication.

\section{Availability of data and materials}

The data that support the findings of this study are available from the corresponding author, N.H., upon reasonable request.

\section{Declarations}

Ethics approval and consent to participate

Not applicable.

\section{Consent for publication}

Written informed consent was obtained from the patient for the publication of this case report.

\section{Competing interests}

There are no conflicts of interest regarding the publication of this paper.

Received: 27 May 2021 Revised: 21 June 2021 Accepted: 19 July 2021 Published online: 28 July 2021

\section{References}

1. Doi M, Morita K, Takeda J, Sakamoto A, Yamakage M, Suzuki T. Efficacy and safety of remimazolam versus propofol for general anesthesia: a multicenter, single-blind, randomized, parallel-group, phase Ilb/III trial. J Anesth. 2020;34:543-53.

2. Doi M, Hirata N, Suzuki T, Morisaki H, Morimatsu H, Sakamoto A. Safety and efficacy of remimazolam in induction and maintenance of general anesthesia in high-risk surgical patient (ASA Class III): results of a multicenter, randomized, double-blind, parallel-group comparative trial. J Anesth. 2020;34:491-501. 
3. Miyake W, Oda Y, Ikeda Y, et al. Electroencephalographic response following midazolam-induced general anesthesia: relationship to plasma and effect-site midazolam concentrations. J Anesth. 2010;24:386-93.

4. Glass PS, Bloom M, Kearse L, et al. Bispectral analysis measures sedation and memory effects of propofol, midazolam, isoflurane, and alfentanil in healthy volunteers. Anesthesiology. 1997;86:836-47.

\section{Publisher's Note}

Springer Nature remains neutral with regard to jurisdictional claims in published maps and institutional affiliations.

\section{Submit your manuscript to a SpringerOpen ${ }^{\circ}$ journal and benefit from:}

- Convenient online submission

- Rigorous peer review

- Open access: articles freely available online

- High visibility within the field

- Retaining the copyright to your article

Submit your next manuscript at $\mathbf{s p r i n g e r o p e n . c o m ~}$ 